\title{
Specific Heatof the Filled Skutterudite Superconductor $\mathrm{LaOs}_{4} \mathrm{As}_{12}$
}

\author{
J. Juraszek, Z. Henkie And T. CichoreK* \\ Institute of Low Temperature and Structure Research, Polish Academy of Sciences, \\ Okólna 2, 50-422 Wrocław, Poland \\ We investigated low-temperature specific heat of the filled skutterudite compound $\mathrm{LaOs}_{4} \mathrm{As}_{12}$ with a transition \\ temperature $T_{c}=3.2 \mathrm{~K}$. A moderate size of a specific-heat jump points at a weak electron-phonon coupling in \\ this material. At $T<T_{c}$, the electronic contribution to the specific heat was found to be at variance with the \\ predictions for an $s$-wave superconductor with one energy gap. Additionally, we observed an upward curvature of \\ the upper critical field near the transition temperature. Both features provide some indications towards multiband \\ superconductivity, although anisotropy effects as a viable cause of the observed anomalies in $\mathrm{LaOs}_{4} \mathrm{As}_{12}$ cannot be \\ excluded. Similarities to a probable two-band superconductor $\mathrm{LaRu}_{4} \mathrm{As}_{12}$ are discussed.
}

DOI: 10.12693/APhysPolA.130.597

PACS/topics: 74.25.Bt, 74.70.Dd

\section{Introduction}

Shortly after the first announcement of the BCS theory of superconductivity [1] it was pointed out that substantial interband scattering can give rise to two-band superconductivity (TBSC) $[2,3]$. To date, much of the research on multiband superconductivity has focused on layered materials with a strongly anisotropic Fermi surface (FS) such as, e.g., $\mathrm{MgB}_{2}$ [4], $\mathrm{NbSe}_{2}$ [5], nonmagnetic borocarbides [6, 7], $\mathrm{Lu}_{2} \mathrm{Fe}_{3} \mathrm{Si}_{5}$ [8], and iron pnictides and chalcogenides $[9,10]$. Anisotropy often leads to similar modifications in single-band superconductors, which is why the distinction of multiband and anisotropy effects is usually difficult, particularly when the temperature dependence of the quantities is examined. In contrast, the field-dependent effects are more often different and thus, more often allow a reliable distinction between different scenarios [11].

In certain two-band superconductors with cubic symmetry, distinct superconducting gaps may reside on different sheets of a weakly anisotropic Fermi surface. Multiband effects most likely coincide with an exotic pairing mechanism in the heavy-fermion superconductor $\mathrm{PrOs}_{4} \mathrm{Sb}_{12}$, as inferred from thermal-conductivity measurements in varying magnetic fields [12, 13]. Furthermore, there are some claims for the observation of twoband superconductivity in its isostructural counterpart $\mathrm{PrRu}_{4} \mathrm{Sb}_{12}$ [13]. Remarkable that $\mathrm{LaRu}_{4} \mathrm{As}_{12}$, adopting the same filled skutterudite crystal structure, displays several features indicative of two-band superconductivity, although a lack of $4 f$ electrons makes its electronic structure less complex. A compelling evidence for more than one energy gap in $\mathrm{LaRu}_{4} \mathrm{As}_{12}$ holds for (i) a nonlinear magnetic field $B$ dependence of the specific heat in the zero-temperature limit, (ii) a positive curvature of the $B_{c 2}(T)$ dependence, and (iii) a deviation of the electronic specific heat from the one-gap $\alpha$ model [14].

\footnotetext{
* corresponding author; e-mail: T.Cichorek@int.pan.wroc.pl
}

Besides $\mathrm{LaFe}_{4} \mathrm{As}_{12}$ and $\mathrm{LaFe}_{4} \mathrm{Sb}_{12}$, all the other Lafilled skutterudite compounds $\mathrm{LaT}_{4} \mathrm{Pn}_{12}(\mathrm{~T}=\mathrm{Ru}, \mathrm{Fe}$ and $\mathrm{Os} ; \mathrm{Pn}=\mathrm{P}, \mathrm{As}$ and $\mathrm{Sb}$ ) display $s$-wave superconductivity. As far as superconductivity of the $\mathrm{LaT}_{4} \mathrm{Pn}_{12}$ skutterudites is concerned, we note a smooth change of lattice dynamics with the average atomic mass without any exceptional difference between the $\mathrm{Os}_{4} \mathrm{As}_{12}, \mathrm{Ru}_{4} \mathrm{As}_{12}$, and $\mathrm{Ru}_{4} \mathrm{Sb}_{12}$ frameworks. Since the phonon spectrum of $\mathrm{LaRu}_{4} \mathrm{As}_{12}$ does not show any evidence for unusual behavior with respect to other La-filled skutterudite superconductors, we have performed a similar study on $\mathrm{LaOs}_{4} \mathrm{As}_{12}$ to elucidate a rare observation of multiband effects in the cubic system. Intriguingly, evidence for multiband order parameters is found in $\mathrm{LaRu}_{4} \mathrm{As}_{12}\left(T_{c}=\right.$ $10.45 \mathrm{~K}, B_{c 2} \approx 10.2 \mathrm{~T}$ ), being the material with enhanced superconducting properties as compared to other skutterudite superconductors.

Physical properties of $\mathrm{LaOs}_{4} \mathrm{As}_{12}$ have been investigated by different groups. All available data consistently point at a conventional $s$-wave BCS superconductivity below $T_{c}=3.2 \mathrm{~K}[15]$, but the specific heat was only briefly examined. The Fermi-surface topology of $\mathrm{LaOs}_{4} \mathrm{As}_{12}$, determined by the de Haas-van Alphen oscillations, was found to be in reasonable agreement with the predictions of band-structure calculations: FS consists of a sphere enclosing a small volume in the center of the Brillouin zone and a rather complex three-dimensional sheet. Both parts of the Fermi surface are strictly disconnected from each other.

\section{Experimental procedure}

Single crystals of $\mathrm{LaOs}_{4} \mathrm{As}_{12}$ were grown using the high-temperature molten-metal-flux procedure detailed in Ref. [16]. Elemental components in a molar ratio La:Os:Cd:As = 1:4:12:48 and with purities better than $99.9 \%$ were placed in a quartz ampoule. A heat treatment consisted of: (i) initial heating to $850^{\circ} \mathrm{C}$ within $24 \mathrm{~h}$ and a dwell time at this temperature of five days; (ii) slow cooling to $750^{\circ} \mathrm{C}$ within two weeks; (iii) final cooling to the room temperature within $24 \mathrm{~h}$. As-grown 
crystals were cleaned in acid to remove residual flux and impurity phases from their surfaces. A residual resistivity as small as $\rho_{0}=1.5( \pm 0.4) \mu \Omega \mathrm{cm}$, a correspondingly large residual resistivity ratio $\rho_{(300 K)} / \rho=\mathrm{RRR}=453$ and well-defined de Haas-van Alphen oscillations underline a high-quality of our $\mathrm{LaOs}_{4} \mathrm{As}_{12}$ single crystals.

Specific heat $C(T)$ for several single crystals with total mass $m=7.5(3) \mathrm{mg}$ was determined with the aid of the thermal-relaxation method utilizing a commercial ${ }^{3} \mathrm{He}$ microcalorimeter (PPMS) down to $0.38 \mathrm{~K}$ and in magnetic fields up to $1 \mathrm{~T}$ aligned parallel to the [111] direction.

\section{Results and discussion}

Figure 1 presents the low-temperature specific heat of $\mathrm{LaOs}_{4} \mathrm{As}_{12}$ as $C(T) / T$ vs. temperature squared. The specific heat was measured at zero magnetic field and in an overcritical field of $B=1 \mathrm{~T}$. Based on the $B=1 \mathrm{~T}$ data, we have estimated the phonon contribution $C_{p h}$ yielding the Debye temperature $\Theta_{\mathrm{D}}=360(2) \mathrm{K}$ and the Einstein temperature $\Theta_{\mathrm{E}}=104(4) \mathrm{K}$, in good agreement with previous estimates $\left(\Theta_{\mathrm{D}}=360 \mathrm{~K}, \Theta_{\mathrm{E}}=99.4 \mathrm{~K}\right)$ [17]. Surprisingly, the Sommerfeld coefficient of the electronic specific heat $\gamma$ was found to be $93 \mathrm{~mJ} \mathrm{~mol}^{-1} \mathrm{~K}^{-2}$, i.e., twice larger than that obtained for a polycrystalline sample $\left(\gamma=49 \mathrm{~mJ} \mathrm{~mol}^{-1} \mathrm{~K}^{-2}\right)$ [17]. Results of careful micro-calorimetric measurements additionally indicate a high purity of our $\mathrm{LaOs}_{4} \mathrm{As}_{12}$ single crystals. Indeed, the residual electronic specific heat as small as $0.9 \mathrm{~mJ} \mathrm{~mol} \mathrm{~K}^{-1} \mathrm{~K}^{-2}$ at $T=0.4 \mathrm{~K}$ points out that less than $1 \%$ of conduction electrons do not form the Cooper pairs in $\mathrm{LaOs}_{4} \mathrm{As}_{12}$. This enables a detailed analysis of a superconducting state of $\mathrm{LaOs}_{4} \mathrm{As}_{12}$.

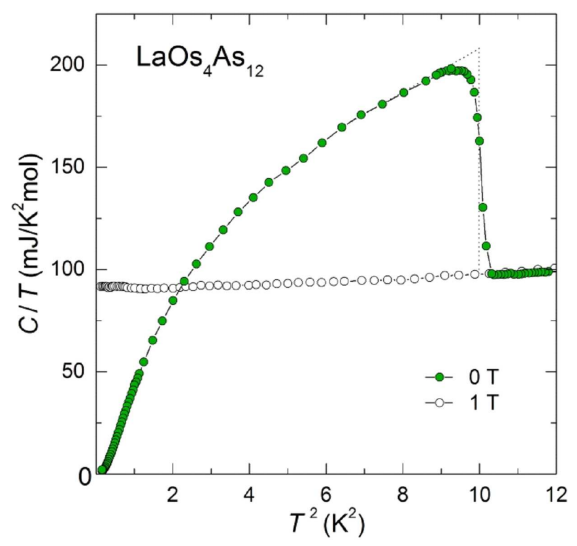

Fig. 1. Low-temperature specific heat for the filled skutterudite compound $\mathrm{LaOs}_{4} \mathrm{As}_{12}$, as $C / T$ vs $T^{2}$, in zero magnetic field and in $B=1 \mathrm{~T}$.

Figure 2 shows the low- $T$ electronic specific heat $C_{e}=$ $C-C_{p h}$ where $C_{p h}$ is the phonon contribution whose temperature dependence was estimated from the normalstate $B=1 \mathrm{~T}$ data. An equal-area construction leaving the entropy unchanged, yields $\Delta C_{e} / \gamma T_{c}=1.19$ that is clearly lower than the weak-coupling BCS prediction $(=1.43)$. We note that a weak electron-phonon coupling in $\mathrm{LaOs}_{4} \mathrm{As}_{12}$ is in striking contrast to a strong coupling in $\mathrm{LaRu}_{4} \mathrm{As}_{12}\left(\Delta C_{e} / \gamma T_{c}=2.26\right)$. However, since superconductivity in $\mathrm{LaRu}_{4} \mathrm{As}_{12}$ is discussed in terms of TBSC, we also consider this possibility for $\mathrm{LaOs}_{4} \mathrm{As}_{12}$. Whereas the one-gap $\alpha$ model correctly describes $C_{e}(T)$ near $T_{c}$, its failure is evident at $T=1.5 \mathrm{~K}$ (cf. dashed line in Fig. 2). Therefore, we have fitted the $C_{e}(T)$ data to the phenomenological two-gap $\alpha$ model [18]. As shown by the solid line, $C_{e}(T)$ for $T \leq T_{c}$ is well reproduced assuming two different gaps $\Delta_{1} / k_{\mathrm{B}} T_{c}=1.87$ and $\Delta_{2} / k_{\mathrm{B}} T_{c}=1.23$ with $40 \%$ and $60 \%$ in relative weight, respectively. Interestingly that a quite similar picture has been applied to certain multiband superconductors, in particular to nonmagnetic borocarbides [7] and iron chalcogenides [10]. Nevertheless, it should be noted that a two-gap correction in the zero-field specific heat of both La-filled arsenide skutterudites is much smaller than the knee-like anomaly in the specific heat of, e.g., $\mathrm{MgB}_{2}$ [4] and $\mathrm{Lu}_{2} \mathrm{Fe}_{3} \mathrm{Si}_{5}$ [8]. Comparison of the residuals of the phonon fit with the residuals of the single gap fit to the derived electronic specific heat are shown in the inset of Fig. 2. Clearly, an unconventional shape of $C_{e}(T)$ at $T<T_{c}$ is not an artefact due to subtraction of the phonon terms.

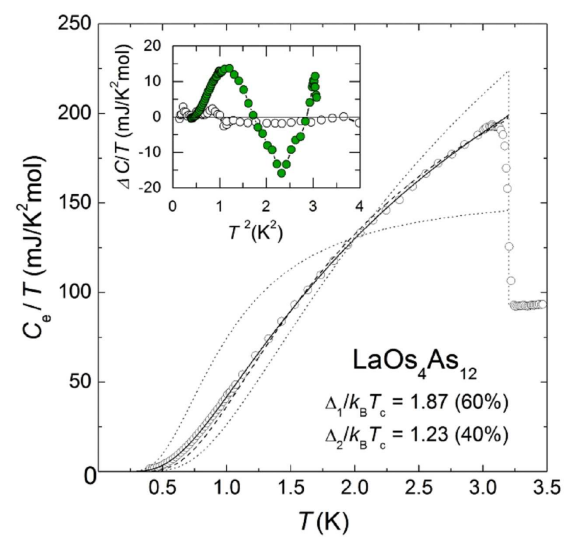

Fig. 2. Low-temperature electronic specific heat of $\mathrm{LaOs}_{4} \mathrm{As}_{12}$. The dashed curve is the result of the onegap $\alpha$ model. The solid line is a fit to the two-gap $\alpha$ model with the partial contribution represented by the two bands (dotted lines). Inset: Comparison of the residuals of the phonon fit (open circles) with the residuals of the single gap fit (solid circles) to the derived electronic specific heat.

Figure 3 shows the $B-T$ phase diagram of $\mathrm{LaOs}_{4} \mathrm{As}_{12}$ as determined from the $C(T)$ measurements. This set of the $B_{c 2}(T)$ data is supplemented by the $C(T)$ results obtained for $\mathrm{LaRu}_{4} \mathrm{As}_{12}$ [15]. Upon lowering temperature below around $0.78 T_{c} \approx 2.5 \mathrm{~K}$ and down to about $0.9 \mathrm{~K}$, the upper critical field increases quasilinearly with the slope $-\mathrm{d} B_{c 2} / \mathrm{d} T=1.11 \mathrm{~T} / \mathrm{K}$, i.e., significantly faster than in the vicinity of $T_{c}$ where $\left.-\mathrm{d} B_{c 2} / \mathrm{d} T\right)_{T_{c}}=$ $0.62 \mathrm{~T} / \mathrm{K}$ was found. As a consequence, a positive curvature emerges whose size is very similar to those observed for $\mathrm{LaRu}_{4} \mathrm{As}_{12}$ [15]. Such upward curvature of the upper critical field was successfully described within an effective 
two-band model based on the sizable coupling between two groups of electrons having different Fermi velocities. However, we note that the characteristic upward curvature of $B_{c 2}$ near the transition temperature may also emerge in anisotropic single-band superconductors [12].

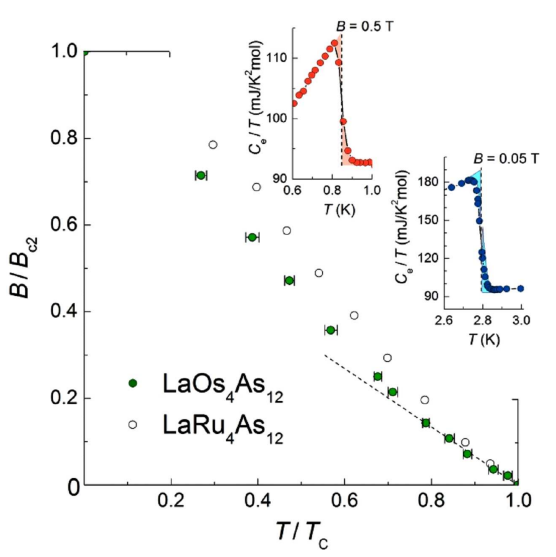

Fig. 3. Magnetic field vs. temperature phase diagram of $\mathrm{LaOs}_{4} \mathrm{As}_{12}$ as obtained from specific-heat measurements (solid circles). Here, the upper critical field of $0.7 \mathrm{~T}$ was assumed. Open circles are data for the closely related compound $\mathrm{LaRu}_{4} \mathrm{As}_{12}$ [15]. The upper critical field was determined from an equal-entropy construction, as exemplified in the upper insets.

Analysis of the zero-field specific-heat data of $\mathrm{LaOs}_{4} \mathrm{As}_{12}$ gives rise to indications towards two-band superconductivity. This is further supported by an unconventional behaviour of the upper critical field near the transition temperature. We note, however, that anisotropy often leads to similar features and hence, a distinction of multiband effects is often difficult, particularly when temperature dependences of the quantities are examined. In contrast, magnetic-field dependent effects are more often different and thus, more often allow a reliable distinction between both phenomena. To this end, we have also measured the specific heat as a function of field down to $T=0.38 \mathrm{~K}$ being the lowest temperature accessible in our experiments. Unfortunately, our preliminary data are too noisy to be analysed. Furthermore, owing $B_{c 2} \approx 0.7 \mathrm{~T}$, a $\gamma(B)$ dependence can be investigated in a rather narrow field range. However, up to about $B=0.1 \mathrm{~T}$ we have observed a linear behaviour of $\gamma(B)$ being much stronger than this expected for an isotropic $s$-wave superconductor. Thus, this fielddependent observation supports multiband superconductivity rather than anisotropy effects as a viable cause of the observed anomalies in $\mathrm{LaOs}_{4} \mathrm{As}_{12}$ adopting the filled skutterudite crystal structure. Forthcoming $C(T)$ experiments on a dilution refrigerator (a base temperature of $0.05 \mathrm{~K}$ ) might help to distinguish between different scenarios.

\section{Conclusions}

We have investigated some superconducting properties of the filled skutterudite compound $\mathrm{LaOs}_{4} \mathrm{As}_{12}$ with
$T_{c}=3.2 \mathrm{~K}$. For this fully gapped superconductor, we have found indications for more than one superconducting energy gap. This holds for a positive curvature of the $B_{c 2}(T)$ dependence in the vicinity of $T_{c}$ and a deviation of the electronic specific heat from the one-gap $\alpha$ model. Thus, $\mathrm{LaOs}_{4} \mathrm{As}_{12}$ with cubic symmetry appears to be an interesting example of a multiband $s$-wave superconductor with a weakly anisotropic Fermi surface.

\section{Acknowledgments}

This work was supported by the Polish National Science Centre within Grant No. 2011/01/B/ST3/05685.

\section{References}

[1] J. Bardeen, L.N. Cooper, J.R. Schrieffer, Phys. Rev. 106, 162 (1957); Phys. Rev. 108, 1175 (1957).

[2] V.A. Moscalenko, Fiz. Met. Metalloved. 8, 503 (1959).

[3] H. Suhl, B.T. Matthias, L.R. Walker, Phys. Rev. Lett. 3, 552 (1959).

[4] F. Bouquet, Y. Wang, I. Sheikin, T. Plackowski, A. Junod, S. Lee, S. Tajima, Phys. Rev. Lett. 89 , 257001 (2002).

[5] E. Boaknin, M.A. Tanatar, J. Paglione, D. Hawthorn, F. Ronning, R.W. Hill, M. Sutherland, L. Taillefer, J. Sonier, S.M. Hayden, J.W. Brill, Phys. Rev. Lett. 90, 117003 (2003).

[6] S.V. Shulga, S.L. Drechsler, G. Fuchs, K.H. Muller, K. Winzer, M. Heinecke, K. Krug, Phys. Rev. Lett. 80, 1730 (1998).

[7] C.L. Huang, J.Y. Lin, C.P. Sun, T.K. Lee, J.D. Kim, E.M. Choi, S.I. Lee, H.D. Yang, Phys. Rev. B 73, 012502 (2006).

[8] Y. Nakajima, T. Nakagawa, T. Tamegai, H. Harima, Phys. Rev.Lett. 100, 157001 (2008).

[9] For a review, see K. Ishida, Y. Nakai, H. Hosono, J. Phys. Soc. Jpn. 78, 062001 (2009).

[10] J. Hu, T.J. Liu, B. Qian, A. Rotaru, L. Spinu, Z.Q. Mao, Phys. Rev. B 83, 134521 (2011).

[11] M. Zehetmayer, Supercond. Sci. Technol. 26, 043001 (2013).

[12] G. Seyfarth, J.P. Brison, M.A. Measson, D. Braithwaite, G. Lapertot, J. Flouquet, Phys. Rev. Lett. 97, 236403 (2006).

[13] R.W. Hill, S. Li, M.B. Maple, L. Taillefer, Phys. Rev. Lett. 101, 237005 (2008).

[14] L. Bochenek, R. Wawryk, Z. Henkie, T. Cichorek, Phys. Rev. B 86, 060511(R) (2012).

[15] I. Shirotani, K. Ohno, C. Sekine, T. Yagi, T. Kawakami, T. Nakanishi, H. Takahashi, J. Tang, A. Matsushita, T. Matsumoto, Physica B 281-282, 1021 (2000)

[16] Z. Henkie, M.B. Maple, A. Pietraszko, R. Wawryk, T. Cichorek, R.E. Baumbach, W.M. Yuhasz, P.C. Ho, J. Phys. Soc. Jpn. 77, Suppl. A, 128 (2008).

[17] K. Matsuhira, C. Sekine, M. Wakeshima, Y. Hinatsu, T. Namiki, K. Takeda, I. Shirotani, H. Sugawara, D. Kikuchi, H. Sato, J. Phys. Soc. Jpn. 78, 124601 (2009).

[18] H. Padamsee, J.E. Neighbor, C.A. Shiffman, J. Low Temp. Phys. 12, 387 (1973). 